\title{
Molecular Characterization of Isolated Chicken Infectious Anemia Viruses in Central China
}

\section{Xinwei Wang}

Henan Agricultural University

Jie Feng

Henan Agricultural University

Ying Liu

Henan Agricultural University

Aijun Sun

Henan Agricultural University

Huayuan Liu

Henan Agricultural University

Xiangru Wang

Henan Agricultural University

Jingjing Wang

Henan Agricultural University

Rui Wang

Henan Agricultural University

Xiaojing Zhu

Henan Agricultural University

Yijie Xu

Henan Agricultural University

Jinglin Qin

Henan Agricultural University

Xia Yang

Henan Agricultural University

Lu Chen

Henan Agricultural University

Guoqing Zhuang ( $\nabla$ gqzhuang2008@163.com )

Henan Agricultural University

\section{Research}


Keywords: Chicken infectious anemia, Epidemiology, Immunosuppressive disease, Live attenuated vaccine, Recombination

Posted Date: May 7th, 2021

DOI: https://doi.org/10.21203/rs.3.rs-459371/v1

License: (c) (1) This work is licensed under a Creative Commons Attribution 4.0 International License. Read Full License 


\section{Abstract}

Background: Chicken infectious anemia (CIA) is an immunosuppressive disease induced by the chicken infectious anemia virus (CIAV) causing heavy economic losses once outbreak. This study conducted a systematic analysis of the epidemiology and pathology of CIA in Henan province, central China.

Methods: A total of 437 clinical tissue samples and 120 poultry disease-related live attenuated vaccines were collected during 2017-2019; of which 45 were positive for CIAV nucleic acid, with a positive rate of $8.08 \%$.

Results: Our results showed that genome sequences similarity among a total of $12 \mathrm{CIAV}$ isolates was high, and ranged from $97.1 \%$ to $99.3 \%$, and their similarity to the vaccine strains Cux-1 and Del-Ros ranged from $97.8 \%$ to $98.6 \%$. However, There were non-synonymous amino acid mutations in the locus of the major capsid proteins VP1, VP2 and VP3 among all isolates. The subsequent sequence analysis indicated that the isolates of $\mathrm{HN}-4$ and $\mathrm{HN}-8$ showed genetic recombination and follow up animal experiments revealed that $\mathrm{HN}-4$ might be a high pathogenic strain.

Conclusions: Our results reveal that both field infection and vaccine contamination promote epidemiology of CIAV in China. Some dominant epidemic viruses have undergone recombination and evolution. This study provides important information for CIAV prevention and control in poultry industry development.

\section{Introduction}

Chicken infectious anemia (CIA) is a viral disease caused by the chicken infectious anemia virus (CIAV). The disease is characterized by aplastic anemia in chickens and lymphatic atrophy [1]. CIAV is a nonencapsulated, symmetrical icosahedral virus particle, with an average diameter of $25 \mathrm{~nm}$. The genome of CIAV is single-stranded, circular, negative-stranded covalently closed DNA, of $\sim 2 \mathrm{~kb}$, which contains three partially overlapping open reading frames (ORF) encoding VP1, VP2 and VP3, respectively [2]. VP1 represents the nucleocapsid protein of CIAV and is the main immunogen, which encodes an arginine-rich polypeptide with a molecular weight of approximately $50 \mathrm{KDa}$ [3]. VP2 is an auxiliary scaffold protein required for CIAV assembly, which helps VP1 to form the correct conformation [4], and both VP1 and VP2 can cooperate with each other to induce an immune response. VP3 is a functional protein of the virus, which is also known as apoptin [5]; studies have found that transfection of the VP3 gene into chicken monocyte cultures, can induce apoptosis, highlighting its importance in CIAV infection [6].

CIAV mainly invades the bone marrow and thymus of the central immune organ, leading to yellowing of the bone marrow and atrophy of the thymus, and can also cause aplastic anemia and immunosuppression [7]. As one of the important poultry diseases, CIAV infection can either directly reduce the protective efficacy of certain vaccines, or enhance pathogenicity of attenuated vaccines, leading to immune failure $[8,9]$. The natural mortality rates form CIAV infection in the clinic is approximately $10 \%-20 \%$. Due to the low mortality rates and hidden infectivity, CIAV has often been 
neglected in clinical settings [10]. However, the latent infection caused chicken immunosuppression, which enhanced the invasion of other opportunist pathogens, including bacteria and viruses, which lead to disorders of chicken growth and development [11]. Significantly, CIAV can transmit both horizontally and vertically. Horizontal transmission occurs in the flock usually from the feces, danders or feathers, mainly through oral infection. More importantly however, vertical infection is caused from breeding eggs, which can easily result in an outbreak of CIA in the offspring. Furthermore, previous studies have highlighted the fact that vertical transmission still exists, even if there is an immune response in infected hens [12].

CIAV infection has spread globally causing severe economic losses. For example, $32 \mathrm{CIAV}$ sequences were identified in 2010 in South Korea [13]. In India, 351 serum samples (86.88\%) were positive in 404 serum samples collected from chicken flocks in 11 poultry farms [14], and in northern Vietnam, there were $74(62.2 \%)$ positive tests in 119 samples collected from 64 farms [15]. In China, CIAV has been detected in the poultry farms of most provinces, and has been successfully isolated both from live vaccines and specific pathogen free (SPF) chickens [16], which may explain the reason for the CIAV contamination found in the field. Interestingly, co-infection of four immunosuppressive pathogens including CIAV has been detected in Shandong Province $[17,18]$. Recently, 1,187 clinical samples from major poultry farms nationwide from 2017 to 2019, have been tested for co-infection with six immunosuppressive pathogens including CIAV [19]. These surveys have confirmed the widespread nature of CIAV in China, which has seriously affected the development of the poultry industry. However, there is a paucity of information relating to CIAV infection in the central region of China. Therefore, an epidemiological investigation of CIA was carried out on samples collected from chicken farms and live attenuated vaccines in the markets of Henan Province, and the genomes of CIAV epidemic strains in this region were systematically analyzed. Furthermore, the pathogenesis of one recombinant strain has been investigated further.

\section{Materials And Methods}

\section{Sample collection}

The tissue samples were collected from chicken flocks produced throughout Henan province, China, by the Poultry Disease Research Institute of Henan Agricultural University. In brief, tissues from suspected chickens containing CIAV were cut into small pieces and place into a sterile tube. Samples were then homogenized three times in phosphate buffer saline (PBS) using small steel balls in an automated tissue grinder. Next, the samples were freeze/thawed three times to encourage the release of the virus. The viral supernatant was isolated by centrifugation at $8000 \mathrm{rpm}$ and then transferred into a new sterile tube. A total of 120 non-CIAV live attenuated vaccines were collected from 24 manufacturers and five different batches for CIAV detection.

\section{Virus amplification}

Marek's disease lymphoblastoid cell (MSB1) (A kind gift from Professor Jun Ji, Nanyang Normal University, Henan province) were cultured in 1640 culture medium with 10\% fetal bovine serum (Solarbio, 
Beijing, China). When the cell confluency reached $5 \times 10^{5} \mathrm{cells} / \mathrm{cm}^{3}$, they were centrifuged at $1000 \mathrm{rpm}$ for 5 min and washed twice with sterile PBS to remove residual serum. Then the CIAV containing supernatant was added for virus adsorption for $1 \mathrm{~h}$. After this step, MSB1 cells were cultured in $5 \mathrm{~mL}$ of 1640 medium containing $2 \%$ fetal bovine serum and When visible cytopathic changes could be seen, the infected cells were passaged blind five times on MSB1 cells, for viral amplification.

\section{NucleicAcid extraction and CIAV detection}

Genomic DNA was extracted from the chicken samples using a commercial kit (TIANamp Genomic DNA

Kit, Tiangen, Beijing, China) and used according to the manufacturer's instructions. Three pairs of primers for amplification of the CIAV genome were designed and synthesized by Primer Premier 5.0 (Sangon Biotech, Shanghai, China). The first primer pair were as follows: forward primer 5'-

GCATTCCGAGTGGTTACTATTCC-3', reverse primer 5'-CGTCTTGCCATCTTACAGTCTTAT-3'; and these primers were expected to produce a predicted amplicon size of $842 \mathrm{bp}$. The second primer pair were as follows: forward primer 5'-CGAGTACAGGGTAAGCGAGCTAAA-3', reverse primer

5'-TGCTATTCATGCAGCGGACTT-3'; and these primers produced a predicted amplicon size of $990 \mathrm{bp}$. The final set of primers were as follows: forward primer 5'-GAAAATGAGACCCGACGAGCAACAG-3', reverse primer

5'-GATTCGTCCATCTTGACTTTCTGTG-3', and these primers produced a predicted amplicon size of 736 bp. The polymerase chain reaction (PCR) reaction was performed in a total volume of $50 \mu \mathrm{L}$, containing $18 \mu \mathrm{L}$ of $2 \times$ Taq Master Mix, $1 \mu \mathrm{L}(10 \mu \mathrm{m} / \mathrm{L})$ of each primer, $25 \mu \mathrm{L}$ distilled water, and $3 \mu \mathrm{L}$ template DNA. The PCR cycling conditions for amplification were as follows: $95^{\circ} \mathrm{C}$ pre-denaturation for $5 \mathrm{~min} ; 95^{\circ} \mathrm{C}$ denaturation for $30 \mathrm{~s}, 60^{\circ} \mathrm{C}$ annealing for $30 \mathrm{~s}$, and $72^{\circ} \mathrm{C}$ extension for $30 \mathrm{~s}$. Amplification was performed over 35 cycles with a final extension at $72^{\circ} \mathrm{C}$ for $10 \mathrm{~min}$. The PCR products were then resolved using $1 \%$ agarose gel electrophoresis, included both positive and negative controls.

\section{Sequencing analysis}

The PCR products were purified with a PCR gel recovery kit and cloned into PMD18-T vectors and sent to Sangon Biotech for sequencing, and then aligned using the BLSAT program on the National Center for Biotechnology Information (NCBI) website (www.ncbi.nlm.nih.gov) to reference strains (Table 1). Next, the MEGA 6 and RDP4 softwares were used to analyze the sequences and recombination of the isolates, which incorporated RDP, GENECONV, BootScan, MaxChi, Chimera, SiScan, Phyl-Pro, LARD And 3Seq.

Table 1. Reference CIAVs for Henan isolates sequence analysis 


\begin{tabular}{|c|c|c|}
\hline Virus strain & Area & GenBank accession number \\
\hline 3-IP60 & Malaysia & AY040632 \\
\hline $82-2$ & Japan & D31965 \\
\hline 98D02512 & USA & AF311892 \\
\hline 704 & Australia & U65414 \\
\hline C369 & Japan & AB046590 \\
\hline CIAVV89-69 & Korea & JF507715 \\
\hline clone34 & Germany & AJ297685 \\
\hline Cux-1* & USA & M55918.1 \\
\hline Del-Ros* & USA & AF313470.1 \\
\hline L14767.1 & USA & L14767.1 \\
\hline RS-BR-15 & Brazil & KY024579.1 \\
\hline SMSC-1 & Malaysia & AF285882 \\
\hline SMSC-IP60 & Malaysia & AF390102.1 \\
\hline TR20 & Japan & $\mathrm{AB} 027470$ \\
\hline $\mathrm{AH} 4$ & China & DQ124936.1 \\
\hline CIAV Mouse & China & KU645525 \\
\hline HN1405 & China & KU645520 \\
\hline HN1504 & China & KU645512 \\
\hline isolate 18 & China Taiwan & KJ728827 \\
\hline JN1503 & China & KU641014 \\
\hline LF4 & China & AY839944.2 \\
\hline LN1402 & China & KU645511 \\
\hline LY-1 & China & KX447636.1 \\
\hline SD15 & China & KX811526 \\
\hline SD22 & China & DQ141673 \\
\hline SD1512 & China & KU645506 \\
\hline SDLY08 & China & FJ172347 \\
\hline TJBD40 & China & AY846844.1 \\
\hline
\end{tabular}




\begin{tabular}{|lll|} 
HA4 & China & DQ124934 \\
\hline HLJ15108 & China & KY486137 \\
GD-K-12 & China & KF224935.1 \\
GD-1-12 & China & JX260426 \\
\hline
\end{tabular}

Note: "*" represents vaccine virus.

\section{Animal experiments}

SPF chickens (Qian yuan hao biotechnology Co, Zhengzhou, China) were used for all animal experiments. SPF chickens were wing-banded upon hatching, and housed in isolators, and randomly sorted into experimental groups. An additional group was used as control. To determine the pathogenic properties of the $\mathrm{NH}-4$ isolate, experimental groups from one-day-old SPF chickens were inoculated with $0.1 \mathrm{ml}$ of HN-4 in the muscle tissue, whereas the chickens in the control group were inoculated with an identical volume of physiological saline. All chickens that had died during the experiment (21 days), or were euthanized at the end of the experiment, were necropsied and examined for CIAV-related pathology.

To evaluate the effect of HN-4 on body weight and lymphoid organs, three chickens in each group were euthanatized at 7, 14 and 21 days post-infection, and their body weight and lymphoid organ weights (thymus and bursa) were measured. The ratio of the lymphoid organs was expressed as the weight of lymphoid organ divided by the body weight for each chicken, multiplied by one hundred.

For hematoxylin-eosin (H\&E) staining, tissues were formalin fixed and paraffin embedded and 6-8 $\mu \mathrm{m}$ thick sections were prepared. H\&E staining was carried out following regular procedure and sections were examined under a microscope.

\section{Data analysis}

The ratio of the lymphoid organs and survival rates were analyzed using Graphpad Prism version 8.0.1 software (GraphPad Software, Inc. La Jolla, CA). Statistical t-test analysis was used for each data point, which represented a triplicate average and a value of $P<0.05$ was considered statistically significant.

\section{Results}

\section{Complete Gene Sequence Analysis of isolated CIAVs}

A total of 41 samples were detected to be CIAV positive from 437 tissue samples collected from June 2017 to December 2019 in Henan province. The overall positive rate was 9.4\% (95\% Cl: 6.8\%-12.5\%). Four CIAV positive vaccines in 120 non-CIAV live attenuated vaccines were detected, and the overall positive rate was $3.33 \%$ (95\% Cl: $0.9 \%-8.3 \%$ ) (Table 2 ). A total of $12 \mathrm{CIAV}$ strains were isolated and identified by PCR from chicken samples and as shown in Fig. 1A, the amplicon sizes were 842 bp, 990 bp and 736 bp, respectively, and this was consistent with prediction. 
Table 2. Organize the survey results and the CIAV test form for attenuated vaccine circulating in the market

\begin{tabular}{|llll|}
\hline Sample type & $\begin{array}{l}\text { Number of samples tested } \\
\text { (parts) }\end{array}$ & $\begin{array}{l}\text { Number of positive } \\
\text { samples (parts) }\end{array}$ & $\begin{array}{l}\text { Positive rate/\% } \\
(95 \% \mathrm{Cl})\end{array}$ \\
\hline $\begin{array}{l}\text { Tissue disease } \\
\text { material }\end{array}$ & 437 & 41 & 9.4 \\
NDV+IB & 53 & 3 & $5.66 \rrbracket 1.2-15.7 \rrbracket$ \\
\hline FPV & 31 & 1 & $3.23 \llbracket 0.1-16.7 \rrbracket$ \\
\hline NDV & 34 & 0 & $0(0-97.5)$ \\
MS & 1 & 0 & $0(0-97.5)$ \\
\hline FPV+ILTV & 1 & 0 & $0(0-97.5)$ \\
\hline
\end{tabular}

The whole genome sequences of $12 \mathrm{CIAV}$ isolates were amplified and aligned to the reference strain. All isolates showed high similarity, ranging from $97.1 \%$ to $99.3 \%$. Compared with $32 \mathrm{CIAV}$ reference strains, four strains ( $\mathrm{HN}-3, \mathrm{HN}-5, \mathrm{HN}-7, \mathrm{HN}-10)$ showed $99 \%$ identity with the field strain isolated from Shandong province, China. The similarity between $\mathrm{HN}-11$ strain and the Guangdong strain GD-1-12 was $99.1 \%$, and $96 \%$ with Guangdong strain GD-K-12. The three strains HN-2, HN-4 and HN-6 had similarities of $98.4 \%$ with the previously isolated HN1405 strain (Fig. S1).

Four strains of CIAV virus ( $\mathrm{HN}-1, \mathrm{HN}-8, \mathrm{HN}-9, \mathrm{HN}-12)$ had a similarity between $98.1 \%$ and $99.7 \%$, of which, $\mathrm{HN}-1$ and $\mathrm{HN}-9$ had the highest similarity. These four isolates are more closely related to the American strain L14767.1, with a similarity of over $98 \%$. Compared to the vaccine strains that have been widely used worldwide, the 12 isolates had high sequence similarity with the Del-Ros vaccine strain, and ranged from $97.8 \%$ to $98.6 \%$, and among which, the $\mathrm{HN}-11$ isolate had the highest similarity with the vaccine strain. The similarity between the isolated strains and the Cux-1 vaccine strain worldwide was less than 98\% (Fig. S1).

A phylogenetic tree, represented by two large branches, was constructed based on the complete genome sequence of 44 reference strains and $12 \mathrm{CIAV}$ isolates from Henan. All of the Henan isolates were on the same large branch, which was closely related to the CIAV strains derived from Asia. After subdivision, the Henan CIAV isolate became located to three different small branches. The four strains HN-3, HN-5, HN-7, and $\mathrm{HN}-10$ however, were on the same branch as the CIAV strains isolated from Shandong and Liaoning. The HN-11 and the Guangdong isolated strain GD-1-12 were on the same small branch. Both the HN-11 and the Guangdong strain, GD-1-12 evolved from the classical attenuated strain C369, isolated from Japan, and the attenuated strain, CIAVV89-69 isolated from Korea. However, the four strains HN-3, HN-5, $\mathrm{HN}-7$, and $\mathrm{HN}-10$ and the CIAV strains isolated from Shandong and Liaoning were closely related to the classical attenuated strain C369 and CIAVV89-69. The three strains HN-2, HN-4 and HN-6 appear on the same branch as the HN1405 strain isolated from Shandong. HN-1, HN-8, HN-9, and HN-12 are on the 
same branch as the HN1504 strain. All of the CIAV strains isolated in Henan are far removed from the internationally prevalent vaccine strain Cux-1, and are not on the small branch with the Del-Ros vaccine strain (Fig. 1B).

\section{Amino acid sequence analysis of the VP1 and VP2 proteins}

Amino acid sequence analysis based on the VP1 sequence (450 aa) showed that, the overall variation in VP1 protein from all of the isolates was $0-3.4 \%$. The similarity in the VP1 amino acid sequence for the $\mathrm{HN}-1$ and $\mathrm{HN}-12$ strains reached $100 \%$, and the sequence similarity with the American strain L14767.1 was very high (Fig. S2). When comparing the VP1 protein amino acid evolutionary tree (Fig. 2A), and the nucleotide sequence evolutionary tree (Fig. 2B), we found that the VP1 nucleotide tree was roughly the same as the whole genome nucleotide evolutionary tree. When we compared the amino acid trees, we found that the three strains, $\mathrm{HN}-3, \mathrm{HN}-4$, and HN-7 were on the same branch as GD-1-12, while the HN-3 and $\mathrm{HN}-7$ isolates were on the same branch as the $\mathrm{HN}-5$, and $\mathrm{HN}-10$ isolates. Finally, the $\mathrm{HN}-4$ isolate was on the same branch as the $\mathrm{HN}-2$, and $\mathrm{HN}-6$ isolates. Therefore, it can be inferred that there may exist site mutations, or sequence recombinations in these strains at the amino acid level. $\mathrm{HN}-1, \mathrm{HN}-8, \mathrm{HN}-9$, and $\mathrm{HN}-12$ still represent a large branch, which is not different from the phylogenetic tree at the nucleotide level.

VP2 is the most conserved CIAV protein and there are no significant differences at the amino acid level between the Henan isolates and the reference strain, and the co-efficiency of variation was less than $2.3 \%$ (Fig. S3). The VP2 nucleotide phylogenetic tree (Fig. 2C) and amino acid phylogenetic tree (Fig. 2D) displayed no obvious phylogenetic pattern. Among them, $\mathrm{HN}-1, \mathrm{HN}-6, \mathrm{HN}-7$ and $\mathrm{HN}-8$ had an obvious developmental trend within the VP2 amino acid evolutionary tree, which may be related to mutations at their amino acid sites.

From the results seen using similarity analyses, the VP3 protein from the Henan isolates and reference strain, are more conservative at the amino acid level with a difference co-efficient of 0-1.7\% (Fig. S4). The nucleotide phylogenetic tree (Fig. 2E) and the amino acid phylogenetic tree (Fig. 2F) of VP3 had no obvious phylogenetic pattern. The $\mathrm{HN}-1$ and $\mathrm{HN}-2$ strains become extended in the amino acid phylogenetic tree, which may be due to the variation in some amino acid sites in these two isolates. Overall, there were no significant differences in apoptotic proteins in CIAV from the Henan isolates and the other isolates.

\section{Analysis of major amino acid sites of VP1, VP2 and VP3 proteins}

Previous studies have confirmed that the hypervariable region in the VP1 sequence is at amino acid position 13, in which amino acids 139 and 144 have an effect on the replication rate and infection efficiency of the virus in infected cells. When the 139th and 144th amino acids of the strain were glutamine (Q), the proliferation rate was significantly slower. In this study, the amino acid sites for the VP1 protein in 12 isolates, main vaccine strains, classical attenuated strains, domestic virulent strains, and previously isolated strains from Henan were all analyzed. Among these, the 12 isolates from Henan, 
and $\mathrm{HN}-1, \mathrm{HN}-8, \mathrm{HN}-9$, and $\mathrm{HN}-12$ ) strains all carried Q139 and Q144, and the other eight CIAV isolates ( $\mathrm{HN}-2, \mathrm{HN}-3, \mathrm{HN}-4, \mathrm{HN}-5, \mathrm{HN}-6, \mathrm{HN}-7, \mathrm{HN}-10$, and $\mathrm{HN}-11$ ) carried the amino acid lysine (K) at position 139 and glutamate (E) at position 144. All the viruses isolated from Henan contained Q, at position 394, suggesting that the isolates from Henan had high pathogenicity. The amino acid sites for the four isolates $\mathrm{HN}-1, \mathrm{HN}-8, \mathrm{HN}-9$, and $\mathrm{HN}-12$ were the same as those of the HN1504 strain on the VP1 protein, which further provides evidence that the four strains ( $\mathrm{HN}-1, \mathrm{HN}-8, \mathrm{HN}-9$, and $\mathrm{HN}-12)$ are derived from the HN1504 strain. Except for the four isolates, there were mutations at position 22 histidine $(H) 22$ asparagine $(\mathrm{N})$ ), position 75 (valine $(\mathrm{V}) 75 \mathrm{I}$ ) and position 125 leucine $(\mathrm{L})$ (isoleucine (I) $125 \mathrm{~L}$ ) in the VP1 protein of the remaining eight strains. Among them, HN-4, HN-6, and HN-8 had an alanine (A) 290 to proline (P) mutation at position 290 , which has never been previously reported. In addition, there is a serine (S) 287 to $\mathrm{N}$ mutation in the HN-4 strain and an S 287 to threonine (T) mutation in the HN-6 strain. However, whether this mutation influences its pathogenicity, needs further investigation (Table 3).

Table 3. Main amino acid positions of VP1 protein 


\begin{tabular}{|c|c|c|c|c|c|c|c|c|c|c|c|c|c|}
\hline \multirow[t]{2}{*}{ Virus strain } & \multicolumn{13}{|c|}{ VP1 amino acid site } \\
\hline & 22 & 75 & 97 & 125 & 139 & 144 & 157 & 287 & 290 & 370 & 376 & 413 & 446 \\
\hline Cux-1 & $\mathrm{H}$ & V & M & 1 & $\mathrm{~K}$ & $\mathrm{D}$ & v & A & A & S & $\mathrm{L}$ & A & $\mathrm{T}$ \\
\hline Del-Ros & . & . & . & . & . & E & . & $S$ & . & G & . & S & G \\
\hline C369 & . & . & . & L & . & E & & $S$ & . & G & 1 & $S$ & S \\
\hline GD-1-12 & . & . & . & L & . & E & $M$ & $S$ & . & G & 1 & S & S \\
\hline HN1405 & . & . & . & L & . & E & . & $S$ & . & A & . & . & S \\
\hline HN1504 & $\mathrm{N}$ & I & L & . & $\mathrm{Q}$ & $\mathrm{Q}$ & . & . & . & $\cdot$ & . & . & S \\
\hline SMSC-IP60 & . & . & . & . & . & E & $M$ & $S$ & . & G & . & . & . \\
\hline SDLY08 & $\mathrm{N}$ & . & . & . & . & E & & $S$ & . & G & . & $S$ & . \\
\hline $\mathrm{HN}-1$ & $\mathrm{~N}$ & I & L & . & $\mathrm{Q}$ & $\mathrm{Q}$ & . & . & . & . & . & . & $S$ \\
\hline $\mathrm{HN}-2$ & . & . & L & L & . & E & $M$ & $S$ & . & A & & . & $S$ \\
\hline $\mathrm{HN}-3$ & . & . & . & L & . & E & $M$ & $S$ & . & G & 1 & . & $S$ \\
\hline $\mathrm{HN}-4$ & . & . & L & L & . & E & $M$ & $\mathrm{~N}$ & $P$ & G & 1 & S & $S$ \\
\hline $\mathrm{HN}-5$ & . & . & . & L & . & E & & $S$ & . & G & 1 & $S$ & $S$ \\
\hline $\mathrm{HN}-6$ & . & . & L & L & . & E & $M$ & $\mathrm{~T}$ & $P$ & A & . & . & $S$ \\
\hline $\mathrm{HN}-7$ & . & . & $\cdot$ & L & & $E$ & $M$ & $S$ & . & G & 1 & S & $S$ \\
\hline HN-8 & $N$ & 1 & L & . & $\mathrm{Q}$ & $\mathrm{Q}$ & & . & $P$ & G & 1 & & $S$ \\
\hline HN-9 & $N$ & I & L & . & $\mathrm{Q}$ & $\mathrm{Q}$ & & . & . & . & . & & $S$ \\
\hline $\mathrm{HN}-10$ & . & . & . & L & . & $E$ & . & $S$ & . & G & 1 & $S$ & $S$ \\
\hline $\mathrm{HN}-11$ & & . & & L & & $E$ & . & $S$ & . & G & 1 & $S$ & $S$ \\
\hline $\mathrm{HN}-12$ & $\mathrm{~N}$ & I & L & . & $\mathrm{Q}$ & $\mathrm{Q}$ & . & . & . & . & . & . & $S$ \\
\hline
\end{tabular}

The amino acid sites of the VP2 and VP3 proteins were relatively conserved with only a few mutated amino acid positions. In VP2, five alternative amino acid mutations were observed: glycine (G) 31 to E mutation at position 31 and alanine (A) 53 to $V$ mutation at position 53 in the $\mathrm{HN}-1$ isolate, a serine (S) 13 to arginine (R) mutation in $\mathrm{HN}-6$ isolate, a lysine (K) 102 to $\mathrm{E}$ mutation in $\mathrm{HN}-7$ isolate, and a $\mathrm{G} 24$ to $\mathrm{E}$ mutation at position 24 in the HN-8 isolate. In VP3, a P 18 to $S$ mutation was observed in HN-1 isolates, and an $\mathrm{A} 54$ to $\mathrm{G}$ mutation was observed in $\mathrm{HN}-2$ isolates (Table 4).

Table 4. Main amino acid positions of VP2 and VP3 proteins 


\begin{tabular}{|c|c|c|c|c|c|c|c|}
\hline \multirow[t]{2}{*}{ Virus strain } & \multicolumn{5}{|c|}{ VP2 amino acid site } & \multicolumn{2}{|c|}{ VP3 amino acid site } \\
\hline & 13 & 24 & 31 & 53 & 102 & 18 & 54 \\
\hline Cux-1 & $\mathrm{S}$ & G & $\mathrm{G}$ & A & K & $\mathrm{P}$ & A \\
\hline Del-Ros & . & . & . & . & . & . & . \\
\hline C369 & . & . & . & . & . & . & . \\
\hline GD-1-12 & . & . & . & . & . & . & . \\
\hline HN1405 & . & . & . & . & . & . & . \\
\hline HN1504 & . & . & . & . & . & . & . \\
\hline SMSC-IP60 & . & . & . & . & . & . & . \\
\hline SDLY08 & . & . & . & . & . & . & . \\
\hline $\mathrm{HN}-1$ & . & . & $\mathrm{E}$ & V & . & S & . \\
\hline $\mathrm{HN}-2$ & . & . & . & . & . & . & $\mathrm{G}$ \\
\hline HN-3 & . & . & . & . & . & . & . \\
\hline $\mathrm{HN}-4$ & . & . & . & . & . & . & . \\
\hline HN-5 & . & . & . & . & . & . & . \\
\hline HN-6 & $\mathrm{R}$ & . & . & . & . & . & . \\
\hline HN-7 & . & . & & . & $E$ & . & . \\
\hline HN-8 & . & $E$ & . & . & . & . & . \\
\hline HN-9 & . & . & . & . & . & . & . \\
\hline HN-10 & . & . & . & . & . & . & . \\
\hline HN-11 & . & . & . & . & . & . & . \\
\hline $\mathrm{HN}-12$ & . & . & . & . & . & . & . \\
\hline
\end{tabular}

\section{Recombination sequence analysis}

Recombination analysis between all isolates and reference strains by RDP 4 software showed that recombination events occurred between different strains. Among them, $\mathrm{HN}-4$ and $\mathrm{HN}-8$ strains may be two potential recombinant strains. The isolate HN-4 may be a potential recombinant strain between the Korean isolate CIAVV89-69 and the Henan isolate HN-2. Bootscan analysis of the sequence of the HN-4 strain and its major and minor parental strains was conducted. The Korean strain CIAVV89-69 was found to be the major parental strain, while the Henan strain $\mathrm{HN}-2$ represented the minor parental strain, and its 
recombination breakpoint was mapped to position 787 (initial breakpoint) and 1707 (termination breakpoint) (Fig. 3A).

Isolate HN-8 may represent a potential recombinant strain between the Brazilian isolate RS-BR-15 and the Henan isolate $\mathrm{HN}-6$. Here, we used Bootscan analysis of the sequence of the $\mathrm{HN}-4$ strain and its major and minor parental strains. We found that the Henan isolate HN- 6 was the major parental strain, while the Brazilian isolate RS-BR-15 was the minor parental strain, and its recombination breakpoint was mapped to position 36 (initial breakpoint) and 2155 (termination breakpoint) (Fig. 3B).

\section{Pathogenesis of HN-4}

To examine the biological characteristics of the CIAV field isolates, we conducted analyses of their pathogenicity by infecting one-day-old SPF chickens with the HN-4 strain. As expected, there were no pathogenic symptoms in the control group. In the experimental group, however, no death was seen from day 1 to day 13, but chickens showed depression, were somnolent, displayed abnormal development and had a significantly lower body weight $(P<0.001)$ (Fig. 4A). Chickens in the experiment group died continuously 14 days post infection, with a total of 10 in 20 deaths seen up until the end of the experiment (21 days) (Fig. 4B). At necropsy, no obvious pathological changes were seen in the liver and kidney, but significant atrophy was seen in the spleen, bursa and thymus, when compared to the control group (Fig. 4C). The tibia of chickens infected with HN-4 was thin and fragile and had yellowish colored bone marrow (Fig. 4D).

To further evaluate the pathogenicity of the HN-4 strain, histological lesions were examined on day 16 in the bursa, thymus, liver, spleen, and kidney. Our findings revealed that there was a significantly reduced number of lymphocytes in the Bursa, Thymus, spleen, and kidney. Whereas liver cells displayed metatropy when compared to the control group (Fig. 4E). These results revealed that HN-4 may be a highly pathogenic strain.

\section{Discussion}

CIAV infection of its natural host, the chicken, results in a low mortality rate, however, CIAV infection does cause important symptoms such as a poor immune response and even immune failure in its host, resulting in the invasion of other secondary pathogens, which can then induce a huge economic loss to the poultry industry [20]. Therefore, it is vital to perform epidemiological and pathological investigations to help control the spread of CIA. We performed a PCR survey of CIAV infection, which was carried out on 437 samples collected from September 2017 to December 2019, with a positive rate of $9.4 \%$ (95\% Cl: $6.8 \%-12.5 \%$ ), confirming the prevalence of $\mathrm{CIA}$ in the field, in the Henan province region of China.

In addition, the safety of a potential vaccine is closely related to the health of chickens [21]. Here, we investigated the extent of CIAV contamination in the live attenuated vaccines sold at market in Henan Province. A molecular epidemiological survey was conducted on vaccine samples from 24 manufacturers, with a total of 120 attenuated vaccines. The results showed that the overall positive rate 
was $3.3 \%$ (95\% Cl: $0.9 \%-8.3 \%$ ), indicating that the necessity for biological products quality control. In the present study, CIAV was mainly tested in the Newcastle disease vaccine, bivalent live attenuated vaccine of Newcastle disease, Avian infectious bronchitis, and Fowlpox live vaccines. Possible CIAV contamination in other important avian diseases, also needs to be further investigated. Unfortunately, we did not isolate or identify live CIAV from these vaccines.

We did however, isolate and identify 12 CIAV strains from clinical chicken samples, and then analyzed their genome sequences. The nucleotide variations among all the isolates ranged from $0.75 \%$ to $3.9 \%$, indicating little difference between these strains and reference strains. The high identity seen in the Henan isolates suggested that these strains are highly conserved. Phylogenetic results revealed that the 12 CIAV strains were in one large branch, most of which were Asian isolates. These isolates were further divided into three branches, where $\mathrm{HN}-3, \mathrm{HN}-5, \mathrm{HN}-7, \mathrm{HN}-10$, and $\mathrm{HN}-11$ were all on the same branch as the Japanese classical attenuated strain C369 and the Korean attenuated strain CIAV 89-69 [22]. Among them, $\mathrm{HN}-11$ was on the same small branch as Guangdong isolate GD-1-12 [23]. The three strains HN-2, $\mathrm{HN}-4$ and $\mathrm{HN}-6$ were on the same branch as the former Henan HN1405 strain, whereas the HN-1, HN-8, $\mathrm{HN}-9$ and HN-12 strains were on the same branch as the former Henan HN1504 strain, indicating that these strains might be the dominant strains in Henan province. However, there were no similar branches seen for the international vaccine strains Cux-1 and Del-Ros [24]. These results indicate that the Henan isolates may be epidemic strains, after a natural recombination and evolutionary event in Asia.

Of the three intact proteins found in the isolates, VP1 protein had the most amino acid site variations. Previous studies have confirmed that higher virulence strains are seen when position 394 in VP1 protein is $Q$, which represents the main genetic determinant of CIAV virulence [25]. In this study, all isolates had Q at this position, indicating that the viral strains have strong virulence. $\mathrm{HN}-4, \mathrm{HN}-6$, and $\mathrm{HN}-8$ have $\mathrm{A} 290$ to P mutations at position 290, which has never been previously reported. However, VP2 and VP3 were relatively conserved, which may not affect their basic functions. These results suggested that the antigenic variation seen in VP1 may be important for virulence.

Recombination is the main source of mutation and the driving force for virus evolution [24]. We tested the 12 isolated CIAV strains and found that there were genetic recombination events in the HN-4 and HN-8 strains. Recombination occurred both in the coding region and the non-coding region, suggesting a higher level of adaptability and epidemic transmission. Importantly, HN-4 infection induced $50 \%$ of death and significant pathological symptoms after inoculation of one-day-old chickens in a 21 day long experiment. These results suggest that $\mathrm{HN}-4$ might be a highly virulent epidemic strain induced by recombination.

\section{Conclusion}

In conclusion, our study has focused on the isolation, identification and analysis of different CIAV strains prevalent in Henan province, and found that there were specific amino acid site variations and gene recombinations. These recombination events may be responsible for the evolution of virulence in the epidemic strains, whose biological characteristics need further investigation. 


\section{Abbreviations}

CIA: Chicken infectious anemia; CIAV: Chicken infectious anemia virus; ORF: Open Reading Frame; MSB1: Marek's disease lymphoblastoid cell; PBS: Phosphate-buffered saline; SPF: Specific pathogen free; PCR: Polymerase chain reaction; NCBI: National Center for Biotechnology Information; H\&E: Hematoxylin-eosin.

\section{Declarations}

\section{Ethics approval and consent to participate}

The animal experiment and procedures used in this study have been approved by the Institutional Animal Care and Use Committee at the Henan Agricultural University (0336 2006-1). All experiments were performed according to the guidelines of the Animal Ethics Procedures and Guidelines of the People's Republic of China.

\section{Consent to publication}

Not applicable.

\section{Availability of data and materials}

All data generated or analyzed during this study are available upon reasonable request.

\section{Competing interest}

The authors declare that there is no conflict of interest.

\section{Funding}

The starting Foundation for Outstanding Young Scientists of Henan Agricultural University (No. 30500690) and the Advanced program of 2020 for returned overseas scholar (No. 30602136). The grants of National Natural Science Foundation of China (No. 31802160)

\section{Authors' contributions}

XWW, JF and GQZ conceived and designed the study. YL, HYL, JJW, performed the experiments. XRW, RW, XJZ, YJX, JLQ, XY, LC analyzed the data. XWW, JF, YL, AJS and GQZ wrote and revised the manuscript. All authors read and approved the final version.

\section{Acknowledgments}

This project was supported by Henan Fengyuan poultry Co., Ltd (Poultry farm disease control and eradication program).

\section{Author details}


${ }^{1}$ College of Veterinary Medicine, Henan Agricultural University, Zhengzhou, 450002, Henan, PR China.

${ }^{2}$ Wolong Animal's Sanitation Administration, Nanyang, Henan, PR China.

* Correspondence:

College of Veterinary Medicine

Henan Agricultural University

Zhengzhou, 450002, Henan, PR China

E-mail: gqzhuang2008@163.com

\section{References}

1. Vaziry A, Silim A, Bleau C, Frenette D, Lamontagne L. Chicken infectious anaemia vaccinal strain persists in the spleen and thymus of young chicks and induces thymic lymphoid cell disorders. Avian Pathol. 2011;40:377-85.

2. Eltahir YM, Qian K, Jin W, Qin A. Analysis of chicken anemia virus genome: evidence of intersubtype recombination. Virol J. 2011;8:512.

3. Lien YY, Huang CH, Sun FC, Sheu SC, Lu TC, Lee MS, Hsueh SC, Chen HJ, Lee MS. Development and characterization of a potential diagnostic monoclonal antibody against capsid protein VP1 of the chicken anemia virus. J Vet Sci. 2012;13:73-9.

4. Peters MA, Jackson DC, Crabb BS, Browning GF. Chicken anemia virus VP2 is a novel dual specificity protein phosphatase. J Biol Chem. 2002;277:39566-73.

5. Feng C, Liang Y, Teodoro JG. The Role of Apoptin in Chicken Anemia Virus Replication. Pathogens 2020, 9(4).

6. Brown AC, Reddy V, Lee J, Nair V. Marek's disease virus oncoprotein Meq physically interacts with the chicken infectious anemia virus-encoded apoptotic protein apoptin. Oncotarget. 2018;9:28910-20.

7. Haridy M, Goryo M, Sasaki J, Okada K. Pathological and immunohistochemical study of chickens with co-infection of Marek's disease virus and chicken anaemia virus. Avian Pathol. 2009;38:469-83.

8. Zhang Y, Cui N, Han N, Wu J, Cui Z, Su S. Depression of Vaccinal Immunity to Marek's Disease by Infection with Chicken Infectious Anemia Virus. Front Microbiol. 2017;8:1863.

9. Su Q, Meng F, Li Y, Zhang Y, Zhang Z, Cui Z, Chang S, Zhao P. Chicken infectious anemia virus helps fowl adenovirus break the protection of maternal antibody and cause inclusion body hepatitishydropericardium syndrome in layers after using co-contaminated Newcastle disease virusattenuated vaccine. Poult Sci. 2019;98:621-8.

10. Hoerr FJ. Clinical aspects of immunosuppression in poultry. Avian Dis. 2010;54:2-15.

11. Erfan AM, Selim AA, Helmy SA, Eriksson P, Naguib MM. Chicken anaemia virus enhances and prolongs subsequent avian influenza (H9N2) and infectious bronchitis viral infections. Vet Microbiol. 
2019;230:123-9.

12. Gimeno IM, Schat KA. Virus-Induced Immunosuppression in Chickens. Avian Dis. 2018;62:272-85.

13. Kim HR, Kwon YK, Bae YC, Oem JK, Lee OS. Molecular characterization of chicken infectious anemia viruses detected from breeder and broiler chickens in South Korea. Poult Sci. 2010;89:2426-31.

14. Bhatt P, Shukla SK, Mahendran M, Dhama K, Chawak MM, Kataria JM. Prevalence of chicken infectious anaemia virus (CIAV) in commercial poultry flocks of northern India: a serological survey. Transbound Emerg Dis. 2011;58:458-60.

15. Huynh LTM, Nguyen GV, Do LD, Dao TD, Le TV, Vu NT, Cao PTB. Chicken infectious anaemia virus infections in chickens in northern Vietnam: epidemiological features and genetic characterization of the causative agent. Avian Pathol. 2020;49:5-14.

16. Li Y, Wang Y, Fang L, Fu J, Cui S, Zhao Y, Cui Z, Chang S, Zhao P: Genomic Analysis of the Chicken Infectious Anemia Virus in a Specific Pathogen-Free Chicken Population in China. Biomed Res Int 2016, 2016:4275718.

17. Meng F, Dong G, Zhang Y, Tian S, Cui Z, Chang S, Zhao P. Co-infection of fowl adenovirus with different immunosuppressive viruses in a chicken flock. Poult Sci. 2018;97:1699-705.

18. Su Q, Wang T, Meng F, Cui Z, Chang S, Zhao P. Synergetic pathogenicity of Newcastle disease vaccines LaSota strain and contaminated chicken infectious anemia virus. Poult Sci. 2019;98:198592.

19. Li X, Zhang K, Pei Y, Xue J, Ruan S, Zhang G. Development and Application of an MRT-qPCR Assay for Detecting Coinfection of Six Vertically Transmitted or Immunosuppressive Avian Viruses. Front Microbiol. 2020;11:1581.

20. Wanganurakkul S, Smith DR, Chintapitaksakul L, Assavalapsakul W. Effective production of recombinant Delta60VP1 chicken anemia virus protein in Escherichia coli and its application to a serodiagnostic indirect ELISA. J Virol Methods. 2020;282:113887.

21. Tseng TY, Liu YC, Hsu YC, Chang PC, Hsieh MK, Shien JH, Ou SC. Preparation of Chicken Anemia Virus (CAV) Virus-Like Particles and Chicken Interleukin-12 for Vaccine Development Using a Baculovirus Expression System. Pathogens. 2019;8:262.

22. Nakamura K, Mase M, Yamamoto Y, Takizawa K, Kabeya M, Wakuda T, Matsuda M, Chikuba T, Yamamoto $Y$, Ohyama T, et al. Inclusion body hepatitis caused by fowl adenovirus in broiler chickens in Japan, 2009-2010. Avian Dis. 2011;55:719-23.

23. Tan C, Wang Z, Lei X, Lu J, Yan Z, Qin J, Chen F, Xie Q, Lin W. Epidemiology, molecular characterization, and recombination analysis of chicken anemia virus in Guangdong province, China. Arch Virol. 2020;165:1409-17.

24. Van Dong H, Tran GTH, Van Nguyen G, Dao TD, Bui VN, Huynh LTM, Takeda Y, Ogawa H, Imai K. Chicken anemia virus in northern Vietnam: molecular characterization reveals multiple genotypes and evidence of recombination. Virus Genes. 2019;55:643-53.

25. Yamaguchi S, Imada T, Kaji N, Mase M, Tsukamoto K, Tanimura N, Yuasa N. Identification of a genetic determinant of pathogenicity in chicken anaemia virus. J Gen Virol. 2001;82:1233-8. 
A
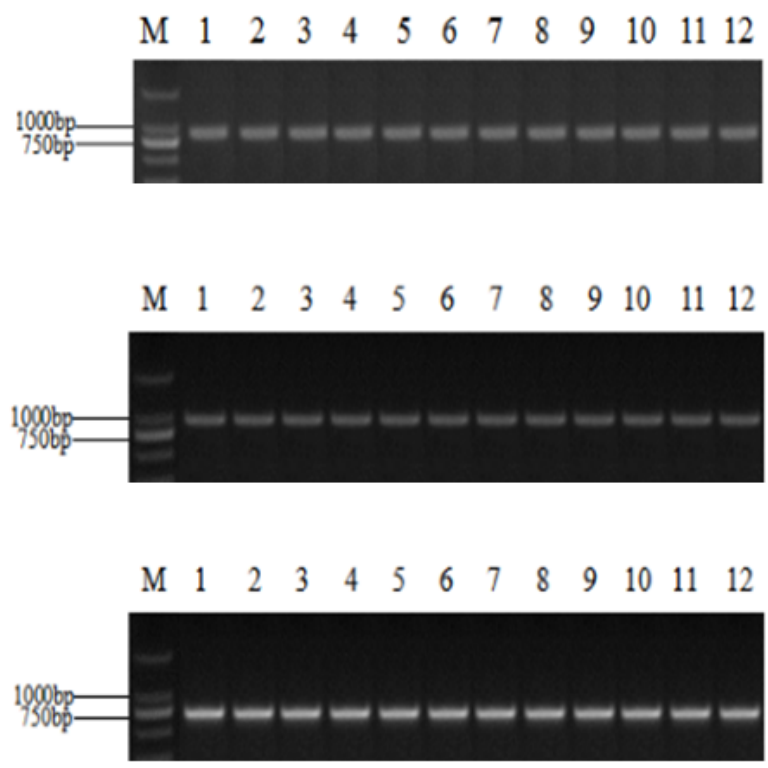

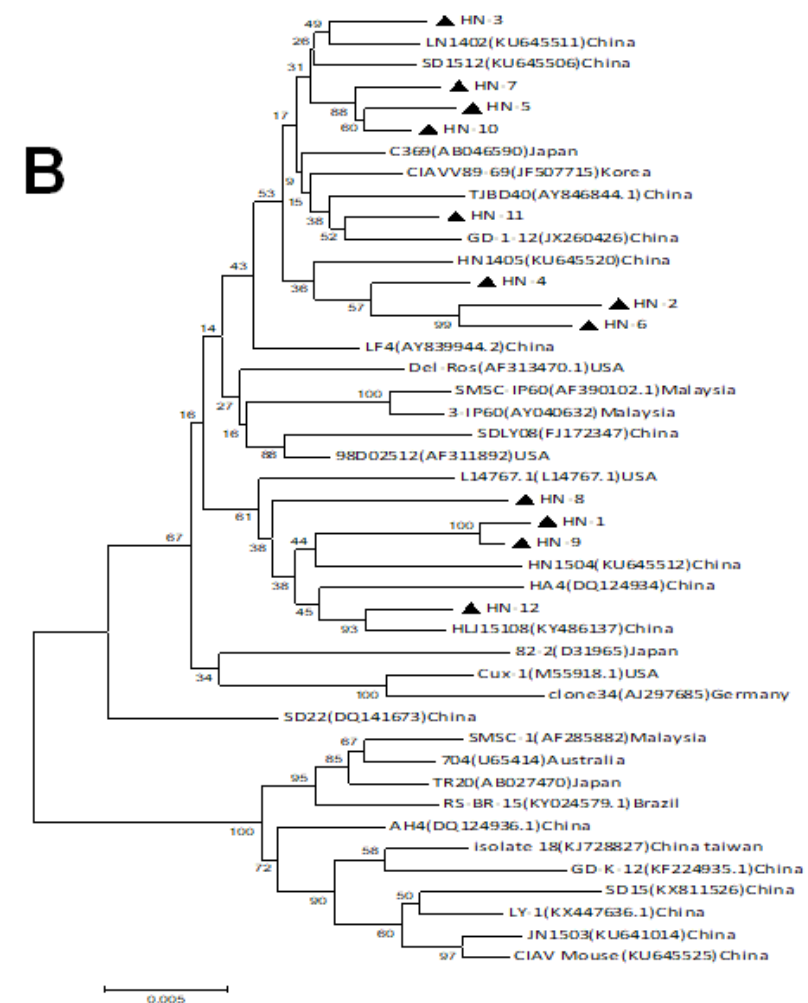

\section{Figure 1}

(A) PCR amplification products of all CIAV strains from Henan. M®DL2000 DNA Marker; Gene fragment 1 of 12 Henan CIAV strains (upper), Gene fragment 2 of 12 Henan CIAV strains (middle), Gene fragment 3 of 12 Henan CIAV strains (lower); (B) Whole genome sequence evolutionary tree analysis. 

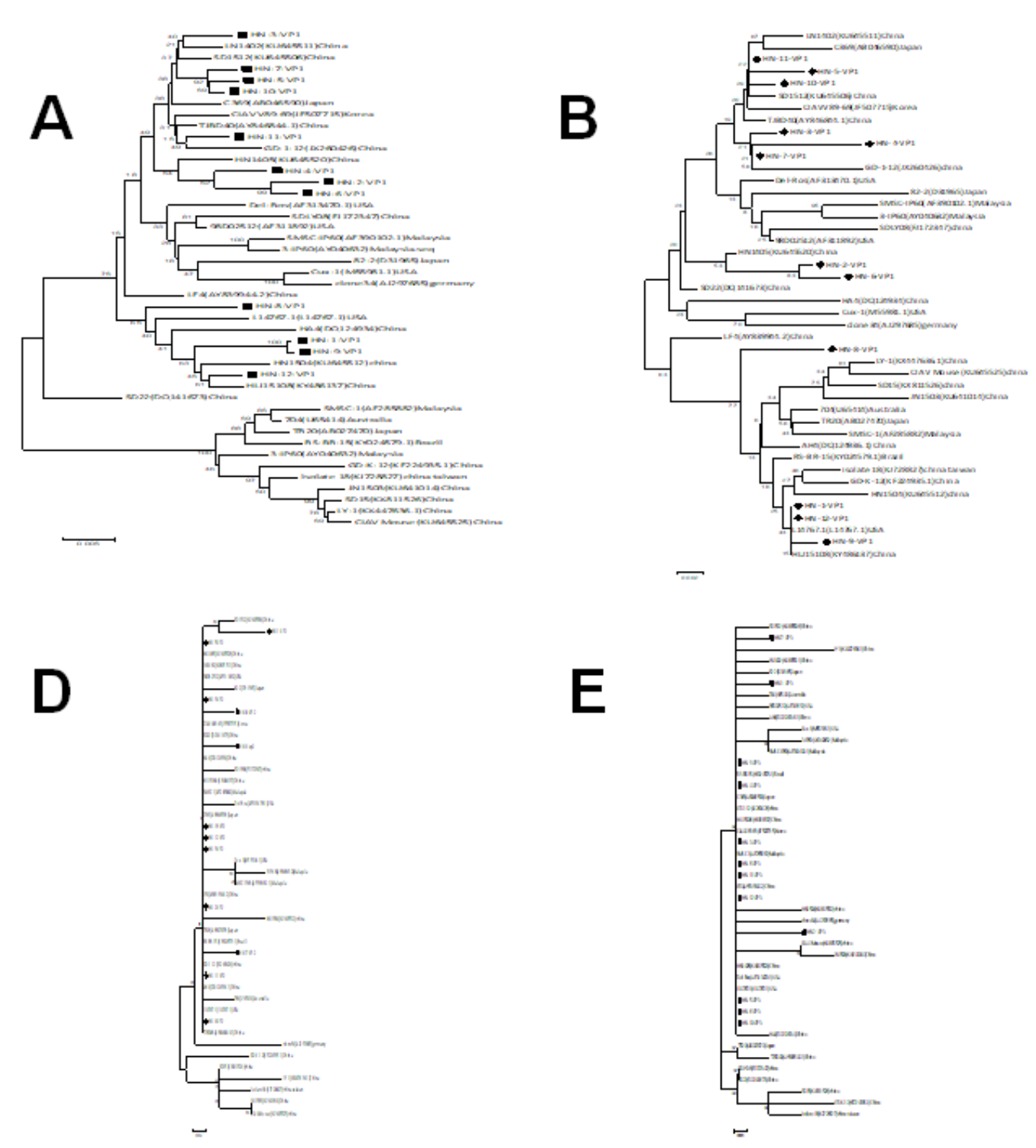
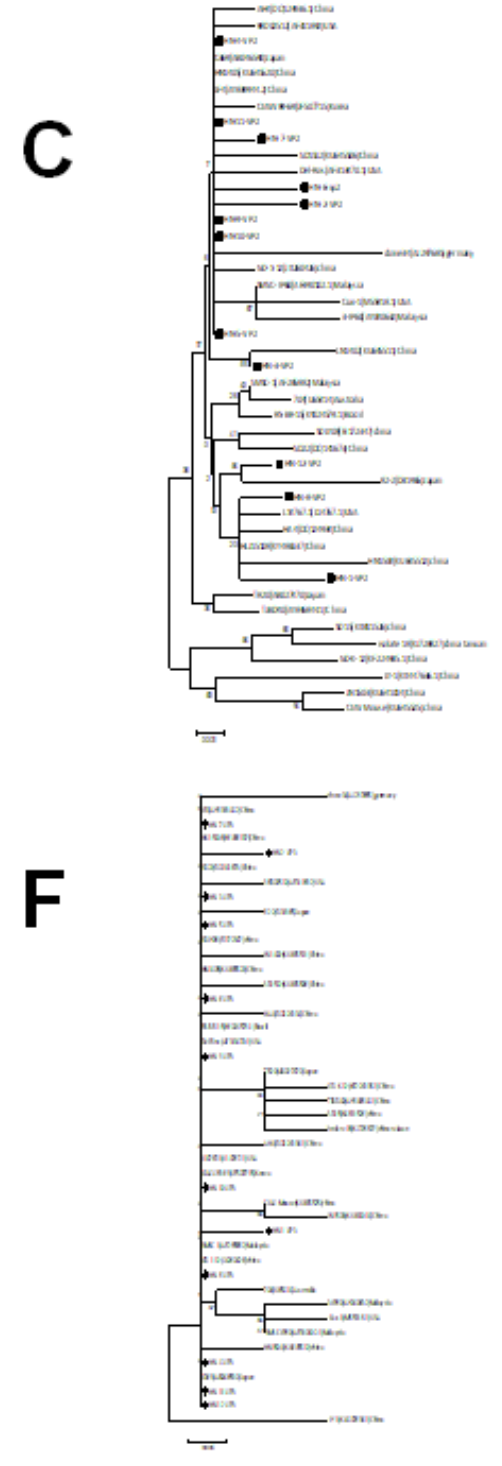

Figure 2

Evolutionary tree analysis of (A) Nucleotide of VP1; (B) Amino acid of VP1; (C) Nucleotide of VP2; (D) Amino acid of VP2; (E) Nucleotide of VP3; (F) Amino acid of VP3. 
A

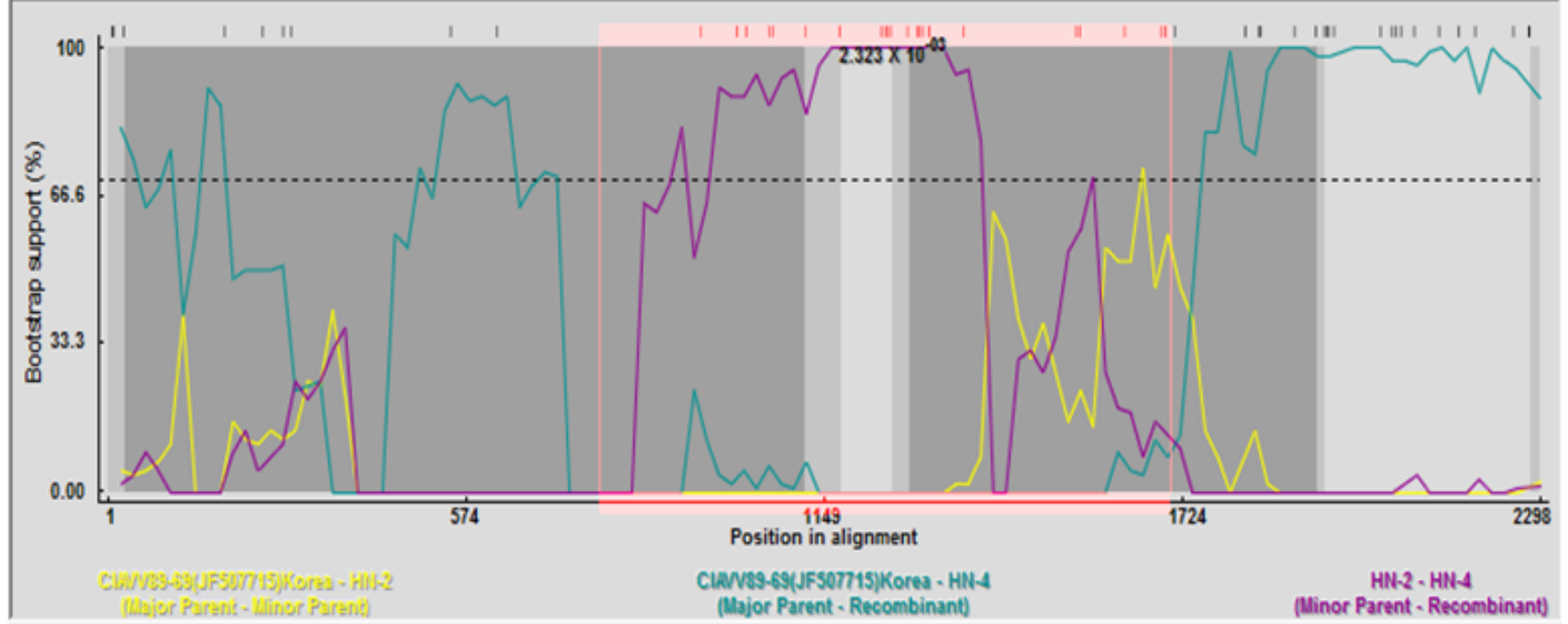

B

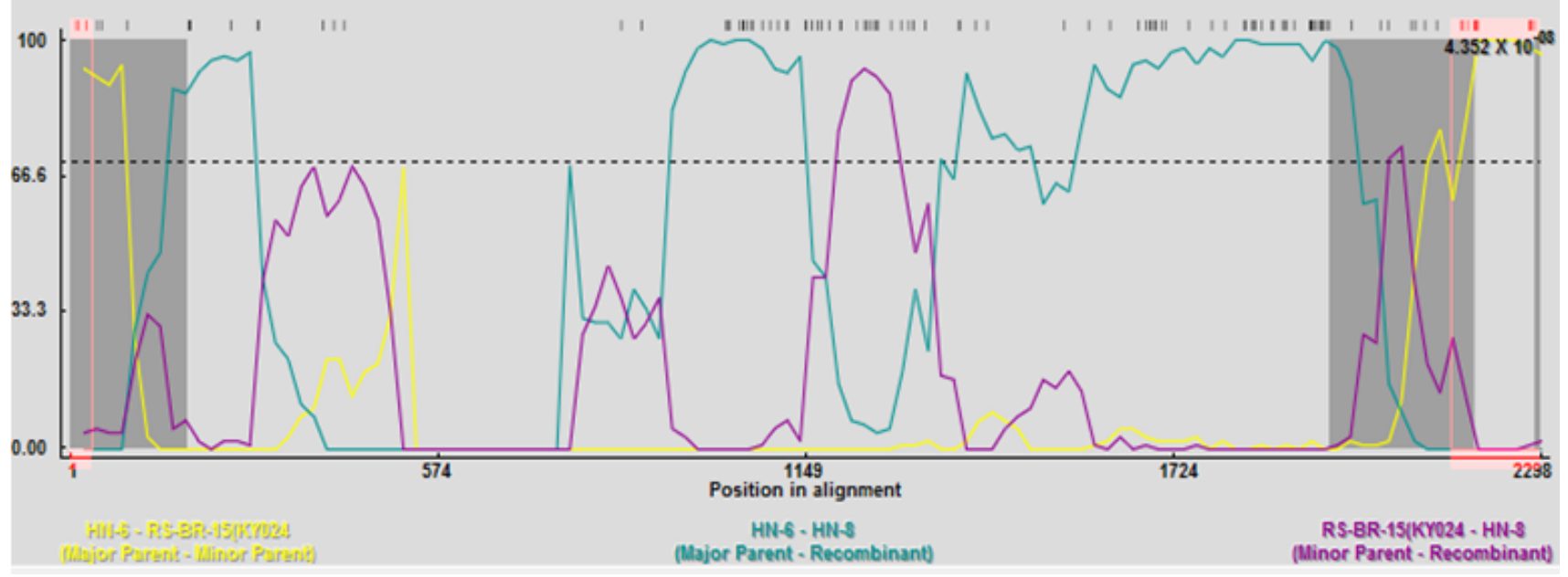

Figure 3

Recombination analysis of the (A) HN-4 strain; and (B) HN-8 strain. 


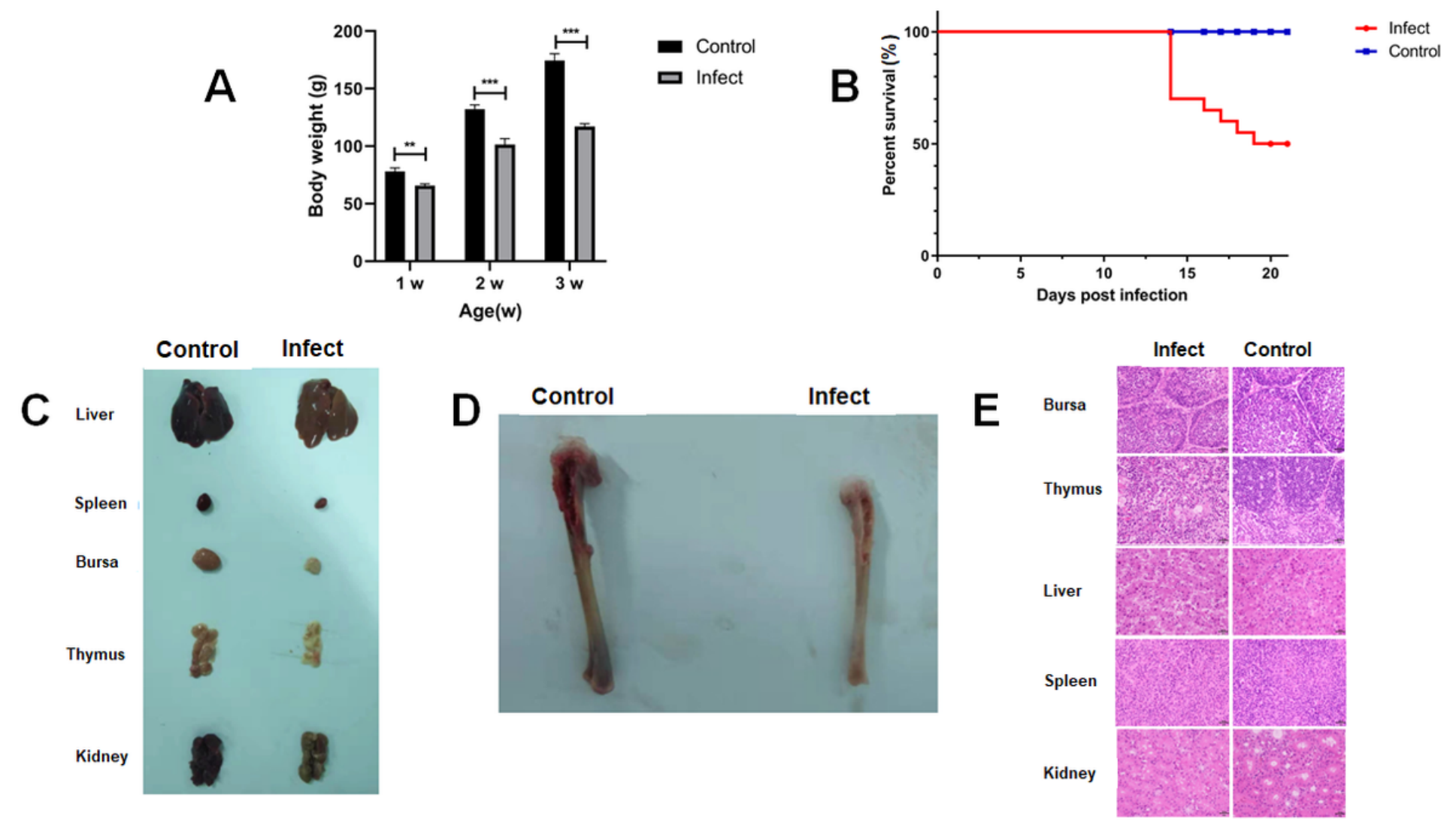

Figure 4

Pathogenic characterization of HN-4. (A) Body weight changes of chickens at different ages (weeks) after $\mathrm{HN}-4$ infection; The statistical difference between groups were examined by student $t$ test. ** and $* \star \star$ represents $p<0.001$. (B) Survival rate of chickens infected with HN-4; (C) Bone marrow changes in chickens infected with HN-4 after 16 days; (D) Pathological changes in various organs from chickens infected with $\mathrm{HN}-4$ after 16 days. (E) Histopathology in different tissues at day 16 post infection (H\&E staining, original magnification $\times 400$ ).

\section{Supplementary Files}

This is a list of supplementary files associated with this preprint. Click to download.

- 2021412SupplementaryMaterials.docx 